\title{
Infant Reflex
}

National Cancer Institute

\section{Source}

National Cancer Institute. Infant Reflex. NCI Thesaurus. Code C81181.

Primitive reflex actions that newborns display in response to specific stimuli. These reflexes are involuntary and most disappear as the infant matures. 